\title{
Analytical Comparisons of Option prices in Stochastic Volatility Models
}

\author{
Vicky Henderson ${ }^{\dagger \ddagger}$
}

August 28, 2002

\begin{abstract}
This paper orders option prices under various well known martingale measures in an incomplete stochastic volatility model. The central result is a comparison theorem which proves convex option prices are decreasing in the market price of volatility risk, the parameter governing the choice of pricing measure. The theorem is applied to order option prices under the minimal martingale, $q$-optimal and minimal entropy measures. This ordering depends on the mean variance tradeoff process whilst the specifics of the volatility dynamics are not important. We illustrate our results by analyzing the Hull and White [15], Heston [13] and Stein and Stein [26] models.
\end{abstract}

Keywords: incomplete markets, stochastic volatility, minimal martingale measure, mean-variance hedging, relative entropy, $q$-optimal measure

\section{Introduction}

Despite the growing number of papers on various approaches to option pricing under stochastic volatility, there are very few concerning comparisons between the many approaches. The aim of this paper is to order option prices under different well known martingale measures in an incomplete stochastic volatility model. We prove a general comparison theorem which says option prices are decreasing in the market price of volatility risk, the parameter which governs the choice of pricing measure. Furthermore, we can identify this parameter under the minimal martingale measure, the $q$-optimal measure and the minimal entropy measure and apply the general result in these cases to obtain an ordering of the option prices under the alternative choices of martingale measure. Our ordering results hold for stochastic volatility models where the correlation between the asset and volatility is zero. The option price results are illustrated by examples using the popular stochastic volatility models of Hull and White [15], Heston [13] and Stein and Stein [26].

\footnotetext{
${ }^{\dagger}$ Nomura Centre for Quantitative Finance, Mathematical Institute, 24-29 St. Giles', Oxford, OX1 3LB. UK. Email: henders1@maths.ox.ac.uk Tel: 44 (0)1865 270502

${ }^{\ddagger}$ I would like to thank David Hobson for his helpful comments
} 
Our results imply that the ordering of option prices under the minimal martingale measure, entropy and $q$-optimal measures hinges on the mean variance tradeoff process. If this is deterministic, then we recover the well known results of Schweizer [22], Pham et al [18] and Schweizer [23] that option prices under variance optimal and minimal entropy are the same as those under the minimal martingale measure. Such models are known in the literature as 'almost complete'. However, if this special case does not hold, then the ordering of option prices depends on whether the mean variance tradeoff process is increasing or decreasing in volatility. This is simple to establish in popular models and is a surprisingly straightforward criteria for ordering option prices. It seems the specific process for the volatility itself does not matter for the ordering. Presumably this feature arises from the assumption of zero correlation between the stock and volatility process.

As an interesting aside, consider the dependence of the market price of volatility risk under the $q$-optimal measure on the parameter $q$. We show this relationship depends on the mean variance tradeoff process. Combining this with the fact that $q \downarrow 1$ gives the minimal entropy market price of volatility risk, enables us to compare option prices under the $q$-optimal and minimal entropy measures.

Although there are many papers on the topic of stochastic volatility, there are not many which compare different pricing approaches. An exception to this is the work of Heath et al [10]. They study quadratic approaches of local risk minimization and mean-variance hedging and make numerical comparisons between option prices and hedging strategies for models with zero correlation. We discuss their results in a later section. The distinction between their paper and this work is that we concentrate on obtaining option price comparisons analytically.

The current paper can be viewed as contributing results on the various approaches to pricing in incomplete markets. Henderson and Hobson [12] examine a similar question for jump diffusion models and order prices based on the jump intensity. Earlier papers by El Karoui et al [4] and others prove convex option prices are ordered in volatility for diffusion (or level dependent volatility) models and some extensions to path dependent payoffs are considered in Henderson [11].

Stochastic volatility models are widely used to overcome some of the deficiencies of the Black Scholes framework. Excess kurtosis in financial time series, leverage effects, and the smiles and skew patterns in implied volatilities all contradict the assumption of constant volatility. The recent paper of Belledin and Schlag [1] tests various stochastic volatility models empirically, and finds these models superior in terms of pricing performance to the Black Scholes model.

Although models including those of Heston [13], Schöbel and Zhu [21] and Scott [24] allow for 'closed form' solutions for option prices via inverse Fourier transforms, these calculations are usually done under the assumption of zero market price of risk, and hence implicitly use the minimal martingale measure. In certain special cases discussed earlier, the option prices under various measures will coincide with the price under the minimal martingale measure, and hence its use is justified. However, this situation is rare, and is not the case 
under most of the original specifications of the models. This simplification to the minimal martingale measure does mean that the incorporation of non-zero correlation is relatively straightforward.

Since stochastic volatility models are incomplete, the direction in mathematical finance has been to use various measures for pricing, see Biagini et al [2], Grandits and Rheinländer [9], Laurent and Pham [17], Heath et al [10], Pham et al [18] in the zero correlation framework and Rheinländer [19] and Hobson [14] with non-zero correlation. The drawback to incorporating a market price of risk is that it is more difficult to calculate explicit forms for the change of measure, particularly for non-zero correlation, although some progress has been made by the last two papers in this area.

The remainder of the paper is structured as follows. Section 2 outlines the form of the stochastic volatility models considered in the paper. The following section describes the three pricing approaches to be considered: minimal martingale measure, $q$-optimal measure and minimal entropy measure and characterizes their market price of volatility risk processes. Section 4 states and proves the main result of the paper, valid for models with correlation zero, relating option price ordering to ordering on the market price of risk. Comparisons of the various market prices of risk are presented in Section 5, including a proof of the result stating the relationship between the $q$-optimal market price of risk and parameter $q$ itself. These comparisons are then combined with the machinery of the main theorem of Section 4 to give the option price result. A further section illustrates these results under three popular stochastic volatility models and a conclusion follows.

\section{Stochastic Volatility models}

This section outlines the form of the stochastic volatility models considered in the paper and will follow Frey [6]. We consider diffusion models of the form:

$$
\frac{d S_{t}}{S_{t}}=v_{t}\left(\mu\left(t, v_{t}\right) d t+d B_{t}\right) \quad d v_{t}=a\left(t, v_{t}\right) d t+b\left(t, v_{t}\right) d W_{t}
$$

where $B, W$ are independent Brownian motions on some probability space $(\Omega, \mathcal{F}, \mathbb{P})$. The filtration $\left(\mathcal{F}_{t}\right)_{t \geq 0}$ is the augmented filtration generated by the Brownian motions. We assume the coefficients of the SDE in (1) satisfy sufficient regularity conditions to ensure the existence of a unique strong solution with the Strong Markov Property, see for example, Karatzas and Shreve [5.2B, $5.4 \mathrm{C}][16]$.

In the model above, we assume zero interest rates for simplicity, so $S$ represents the discounted price of the asset and $v$ models the volatility of $S$, but is not traded. By convention, we take $b\left(t, v_{t}\right) \geq 0$ throughout.

Most of the popular models in the literature fit into this class, including the Stein and Stein [26], Heston [13] and Hull and White [15] models. We consider only the correlation zero case in our analysis, which is a common restriction made by papers on the topic of optimal martingale measures.

To preclude arbitrage, we assume $S$ admits an equivalent local martingale measure $\mathbb{Q}$. Mild conditions on the coefficients guarantee existence of such a 
measure. If $\mathcal{Q}$ denotes the set of such measures then we assume $\mathcal{Q} \neq \emptyset$ and we must choose one of these possible measures to price with. Since $v$ is not traded, the market is incomplete.

A probability measure $\mathbb{Q} \in \mathcal{Q}$ equivalent to $\mathbb{P}$ on $\mathcal{F}_{T}$ is a local martingale measure for $S$ on $\mathcal{F}_{T}$ if and only if there is a progressively measurable process $\lambda=\left(\lambda_{t}\right)_{0 \leq t \leq T}$ with $\int_{0}^{T} \lambda_{s}^{2} d s<\infty \mathbb{P}$ a.s. such that the local martingale $\left(Z_{t}\right)_{0 \leq t \leq T}$ with

$$
Z_{t}=\exp \left(-\int_{0}^{t} \mu\left(u, v_{u}\right) d B_{u}-\frac{1}{2} \int_{0}^{t} \mu\left(u, v_{u}\right)^{2} d u-\int_{0}^{t} \lambda_{u} d W_{u}-\frac{1}{2} \int_{0}^{t} \lambda_{u}^{2} d u\right)
$$

satisfies $\mathbb{E} Z_{T}=1$ and $Z_{T}=\frac{d \mathbb{Q}}{d \mathbb{P}}$ on $\mathcal{F}_{T}$. Thus if $Z_{t}$ is of form (2), $S$ is a $\mathbb{Q}$-local martingale, and if $Z$ is a true $\mathbb{P}$-martingale, $\mathbb{Q}$ is a probability measure.

Note the process $K_{t}=\int_{0}^{t} \mu\left(u, v_{u}\right)^{2} d u$ is often called the mean variance tradeoff process in the literature.

Girsanov's theorem gives two independent $\mathbb{Q}$-Brownian motions $B^{\mathbb{Q}}$ and $W^{\mathbb{Q}}$ defined by

$$
B_{t}^{\mathbb{Q}}=B_{t}+\int_{0}^{t} \mu\left(u, v_{u}\right) d u
$$

and

$$
W_{t}^{\mathbb{Q}}=W_{t}+\int_{0}^{t} \lambda_{u} d u
$$

Under $\mathbb{Q} \in \mathcal{Q}, S$ and $v$ satisfy

$$
\frac{d S_{t}}{S_{t}}=v_{t} d B_{t}^{\mathbb{Q}} \quad d v_{t}=\left[a\left(t, v_{t}\right)-\lambda_{t} b\left(t, v_{t}\right)\right] d t+b\left(t, v_{t}\right) d W_{t}^{\mathbb{Q}} .
$$

Sin [25] gives conditions for the solution $S$ in (3) to be a true martingale.

An option with payoff $h\left(S_{T}\right)$ (we consider $h$ convex) is priced in this model by taking the expectation under any $\mathbb{Q} \in \mathcal{Q}$, ie

$$
\mathbb{E}^{\mathbb{Q}} h\left(S_{T}\right)
$$

Selecting a particular $\mathbb{Q}$ is equivalent to choosing a market price of volatility risk $\lambda_{t}$. We examine various choices in the next section of the paper.

\section{Some Particular Choices of Martingale measure and characterizing the market price of risk}

In this paper we will compare option prices under three different measures $\mathbb{Q} \in \mathcal{Q}$ : the minimal martingale measure of Follmer and Schweizer [5] (also known as local risk minimization), the $q$-optimal measure (of which the variance optimal measure is a special case, see Laurent and Pham [17]) and the minimal entropy measure, see Frittelli [7].

Each method has its advantages and disadvantages. The quadratic approaches of the minimal martingale measure and variance optimal measure are tractable, but are symmetric criterion and treat profits and losses equivalently. 
Heath et al [10] point out that for some models, there is a critical time horizon $T_{0}$ such that the minimal martingale measure exists before this time, but its density is not $p$-integrable after $T_{0}$. A justification for using the minimal entropy measure is its link to some asymptotic exponential utility indifference price, see Frittelli [7].

This section outlines each of these measures and identifies the market price of volatility risk $\lambda$ in each case.

\subsection{Minimal Martingale Measure}

Under the minimal martingale measure, $\mathbb{Q}^{(m)}, S$ is a $\mathbb{Q}^{(m)}$-martingale and every $\mathbb{P}$ martingale orthogonal to the martingale part of $S$ remains a martingale under $\mathbb{Q}^{(m)}$. This results in the market price of risk, $\lambda^{(m)}$ being set to zero and $W^{\mathbb{Q}}$ equals $W$. Thus $\mathbb{Q}^{(m)}$ is minimal in the sense that it preserves the martingale structure as much as possible, whilst making $S$ a martingale.

From (2) we get

$$
Z_{t}=\exp \left(-\int_{0}^{t} \mu\left(u, v_{u}\right) d B_{u}-\frac{1}{2} \int_{0}^{t} \mu\left(u, v_{u}\right)^{2} d u\right) .
$$

For $Z_{T}$ be a true $\mathbb{P}$-martingale, the Novikov condition $\mathbb{E} \exp \left(\frac{1}{2} K_{T}\right)<\infty$ is sufficient, where we recall $K_{T}=\int_{0}^{T} \mu\left(u, v_{u}\right)^{2} d u$. This is straightforward if $K_{T}$ is deterministic. In other models, this may hold for a sufficiently short time horizon, see Heath et al [10].

Under $\mathbb{Q}^{(m)}$ the model in (3) becomes:

$$
\frac{d S_{t}}{S_{t}}=v_{t} d B_{t}^{\mathbb{Q}^{(m)}} \quad d v_{t}=a\left(t, v_{t}\right) d t+b\left(t, v_{t}\right) d W_{t}^{\mathbb{Q}^{(m)}}
$$

with $B_{t}^{\mathbb{Q}^{(m)}}=B_{t}+\int_{0}^{t} \mu\left(u, v_{u}\right) d u$ and $W_{t}^{\mathbb{Q}^{(m)}}=W_{t}$.

\subsection{The $q$-optimal measure}

The $q$-optimal measure $\mathbb{Q}^{(q)}$ (for $q>1$ ) corresponds to the minimum over $\lambda$ of

$$
\mathbb{E}\left[\left(\frac{d \mathbb{Q}}{d \mathbb{P}}\right)^{q}\right]
$$

The variance-optimal measure $\mathbb{Q}^{(2)}$ corresponds to the choice $q=2$, and is the unique measure in those with square integrable density minimizing (5). Existence is dealt with in Delbaen and Schachermayer [3], Gourieroux et al [8] and Rheinländer and Schweizer [20] for continuous processes.

Hobson [14] characterizes the $q$-optimal measure $\mathbb{Q}^{(q)}$ for models of the form (1). The market price of risk for the $q$-optimal measure is given by

$$
\lambda^{(q)}\left(t, v_{t}\right)=b\left(t, v_{t}\right) g^{\prime}\left(t, v_{t}\right)
$$

where $g\left(u, v_{u}\right)$ solves

$$
\frac{q}{2} \mu\left(u, v_{u}\right)^{2}-\frac{1}{2} b\left(u, v_{u}\right)^{2}\left(g^{\prime}\right)^{2}+a\left(u, v_{u}\right) g^{\prime}+\frac{1}{2} b\left(u, v_{u}\right)^{2} g^{\prime \prime}+\dot{g}=0
$$


with $g(T, v)=0$. Using Feynman-Kac gives

$$
g(t, v)=-\log \mathbb{E}\left[\exp \left(-\frac{q}{2} \int_{t}^{T} \mu\left(u, v_{u}\right)^{2} d u\right) \mid v_{t}=v\right]
$$

where under the original measure $\mathbb{P}, d v_{t}=a\left(t, v_{t}\right) d t+b\left(t, v_{t}\right) d W_{t}$. We assume sufficient regularity conditions on the coefficients so Itô's formula can be applied to $g$, see for example Laurent and Pham [17]. For the special case $q=2$, the variance-optimal measure, this Feynman-Kac representation is given in Laurent and Pham [17].

Under $\mathbb{Q}^{(q)}$, the dynamics in $(3)$ become

$$
\frac{d S_{t}}{S_{t}}=v_{t} d B_{t}^{\mathbb{Q}^{(q)}} \quad d v_{t}=\left[a\left(t, v_{t}\right)-b\left(t, v_{t}\right)^{2} g^{\prime}\left(t, v_{t}\right)\right] d t+b\left(t, v_{t}\right) d W_{t}^{\mathbb{Q}^{(q)}}
$$

with $B_{t}^{\mathbb{Q}^{(q)}}=B_{t}+\int_{0}^{t} \mu\left(u, v_{u}\right) d u$ and $W_{t}^{\mathbb{Q}^{(q)}}=W_{t}+\int_{0}^{t} b\left(u, v_{u}\right) g^{\prime}\left(u, v_{u}\right) d u$.

\subsection{Minimal Entropy Measure}

Our third choice of measure $\mathbb{Q}$ is the minimal relative entropy measure, see Frittelli [7]. The relative entropy is defined by

$$
I_{\mathbb{P}}(\mathbb{Q})=\mathbb{E}\left(\frac{d \mathbb{Q}}{d \mathbb{P}} \ln \frac{d \mathbb{Q}}{d \mathbb{P}}\right)=\mathbb{E}^{\mathbb{Q}}\left(\ln \frac{d \mathbb{Q}}{d \mathbb{P}}\right)
$$

and the minimal entropy measure $\mathbb{Q}^{(E)}$ is found by minimizing $I_{\mathbb{P}}(\mathbb{Q})$ over the space of equivalent martingale measures for $S$. Rheinländer [19] gives a necessary and sufficient condition for the existence of an equivalent measure with finite relative entropy. A result of Frittelli [7] then guarantees the minimal entropy measure exists.

Recently, Rheinländer [19] outlined a method to find the minimal entropy measure $\mathbb{Q}^{(E)}$ in a stochastic volatility model. Hobson [14] extended and developed this approach to give an explicit characterization. The market price of risk for the entropy measure is given as

$$
\lambda^{(E)}\left(t, v_{t}\right)=b\left(t, v_{t}\right) f^{\prime}\left(t, v_{t}\right)
$$

where $f(t, v)$ is given by

$$
f(t, v)=-\log \mathbb{E}\left[\exp \left(-\frac{1}{2} \int_{t}^{T} \mu\left(u, v_{u}\right)^{2} d u\right) \mid v_{t}=v\right]
$$

Remark 1 We can immediately see that $f(t, v)$ can be obtained by setting $q=1$ in (8) so $\lambda^{(E)}\left(t, v_{t}\right)=\lim _{q \downarrow 1} \lambda^{(q)}\left(t, v_{t}\right)$.

Under $\mathbb{Q}^{(E)}$, the dynamics in $(3)$ become

$$
\frac{d S_{t}}{S_{t}}=v_{t} d B_{t}^{\mathbb{Q}^{(E)}} \quad d v_{t}=\left[a\left(t, v_{t}\right)-b\left(t, v_{t}\right)^{2} f^{\prime}\left(t, v_{t}\right)\right] d t+b\left(t, v_{t}\right) d W^{\mathbb{Q}^{(E)}}
$$

with $B_{t}^{\mathbb{Q}^{(E)}}=B_{t}+\int_{0}^{t} \mu\left(u, v_{u}\right) d u$ and $W_{t}^{\mathbb{Q}^{(E)}}=W_{t}+\int_{0}^{t} b\left(u, v_{u}\right) f^{\prime}\left(u, v_{u}\right) d u$. 
Remark 2 Before continuing, it is worth noting that in a continuous model where $K_{T}$, the mean variance tradeoff process, is deterministic it is well known that the variance optimal and minimal entropy measures collapse to the minimal martingale measure. In the variance optimal case, this can be found in Schweizer [22] and Pham et al [18]. We recover and extend this result later for q-optimal measures, see Corollary 12. The entropy result can be found in Schweizer [23]. Thus in this case, there is no need for comparison, as option prices will be equal under the three regimes. However, if this condition does not hold, there are no systematic methods to order the prices. This is the question we address.

\section{The General Option Price Ordering Result}

We now prove our main general ordering result which says that convex option prices are decreasing in the market price of risk parameter $\lambda$. This result is proved via coupling. It is here that we need to assume zero correlation between the asset price and volatility process.

Theorem 3 Consider stochastic volatility models of the form (1). Now consider two market price of risk processes $\lambda(t, v)$ and $\gamma(t, v)$ corresponding to measures $\mathbb{Q}^{\lambda}$ and $\mathbb{Q}^{\gamma}$. The volatility processes under these two measures are given by

$$
d v_{t}^{\lambda}=\left[a\left(t, v_{t}^{\lambda}\right)-\lambda\left(t, v_{t}^{\lambda}\right) b\left(t, v_{t}^{\lambda}\right)\right] d t+b\left(t, v_{t}^{\lambda}\right) d W_{t}^{\mathbb{Q}^{\lambda}}
$$

where $W^{\mathbb{Q}^{\lambda}}$ is a $\mathbb{Q}^{\lambda}$-Brownian motion and

$$
d v_{t}^{\gamma}=\left[a\left(t, v_{t}^{\gamma}\right)-\gamma\left(t, v_{t}^{\gamma}\right) b\left(t, v_{t}^{\gamma}\right)\right] d t+b\left(t, v_{t}^{\gamma}\right) d W_{t}^{\mathbb{Q}^{\gamma}}
$$

where $W^{\mathbb{Q}^{\gamma}}$ is a $\mathbb{Q}^{\gamma}$-Brownian motion. Suppose that in each case $v$ is a non-

negative process. The stock price processes are given by $\frac{d S_{t}^{\lambda}}{S_{t}^{\lambda}}=v_{t}^{\lambda} d B_{t}^{\mathbb{Q}^{\lambda}}$ and $\frac{d S_{t}^{\gamma}}{S_{t}^{\gamma}}=v_{t}^{\gamma} d B_{t}^{\mathbb{Q}^{\gamma}}$ where $B^{\mathbb{Q}^{\lambda}}$ and $B^{\mathbb{Q}^{\gamma}}$ are $\mathbb{Q}^{\lambda}, \mathbb{Q}^{\gamma}$-Brownian motions respectively. Then given $\lambda(t, v) \geq \gamma(t, v) \forall t, v \in \mathbb{R}_{+}$;

$$
\mathbb{E}^{\mathbb{Q}^{\lambda}} h\left(S_{T}^{\lambda}\right) \leq \mathbb{E}^{\mathbb{Q}^{\gamma}} h\left(S_{T}^{\gamma}\right)
$$

for convex $h$.

Remark 4 We obtain an ordering on the option prices under two alternative stochastic volatility models, from an ordering on the market price of volatility risk. Option prices are higher in the model with the lower market price of risk parameter.

Remark 5 Note the Theorem as stated only holds for non-negative volatility processes $v$.

Proof: We have measures $\mathbb{Q}^{\lambda}$ and $\mathbb{Q}^{\gamma}$ with volatility processes given by $v^{\lambda}$ and $v^{\gamma}$ defined in (14) and (15). We define a new probability space $\hat{\mathbb{Q}}$ under 
which $\hat{B}, \hat{W}$ are independent Brownian motions. Denote by $\hat{\mathbb{E}}$ expectation under measure $\hat{\mathbb{Q}}$.

Under $\hat{\mathbb{Q}}$ we may write

$$
d \hat{v}_{t}^{\lambda}=\left[a\left(t, \hat{v}_{t}^{\lambda}\right)-\lambda\left(t, \hat{v}_{t}^{\lambda}\right) b\left(t, \hat{v}_{t}^{\lambda}\right)\right] d t+b\left(t, \hat{v}_{t}^{\lambda}\right) d \hat{W}_{t}
$$

where the law of $\hat{v}^{\lambda}$ under $\hat{\mathbb{Q}}$ is the same as the law of $v^{\lambda}$ under $\mathbb{Q}^{\lambda}$. Also write under $\hat{\mathbb{Q}}$

$$
d \hat{v}_{t}^{\gamma}=\left[a\left(t, \hat{v}_{t}^{\gamma}\right)-\gamma\left(t, \hat{v}_{t}^{\gamma}\right) b\left(t, \hat{v}_{t}^{\gamma}\right)\right] d t+b\left(t, \hat{v}_{t}^{\gamma}\right) d \hat{W}_{t}
$$

and set $\hat{v}_{0}^{\lambda}=\hat{v}_{0}^{\gamma}$. Now introduce time changes $A_{t}^{\lambda}=\int_{0}^{t}\left(\hat{v}_{u}^{\lambda}\right)^{2} d u$ and $A_{t}^{\gamma}=$ $\int_{0}^{t}\left(\hat{v}_{u}^{\gamma}\right)^{2} d u$.

We first show that there is an ordering $A_{T}^{\lambda} \leq A_{T}^{\gamma}$. A sufficient condition for this is $\hat{v}_{t}^{\lambda} \leq \hat{v}_{t}^{\gamma}$, since $v$ is non-negative. Given $\lambda(t, v) \geq \gamma(t, v): \forall t, v \in \mathbb{R}_{+}$, a standard stochastic comparison theorem of Karatzas and Shreve [16, 5.2, Proposition 2.18] can be applied here to give $\hat{v}_{t}^{\lambda} \leq \hat{v}_{t}^{\gamma}$.

We now show that this ordering on the time changes is sufficient to prove the ordering on option prices. Given a $\hat{\mathbb{Q}}$-Brownian motion $\hat{B}$, define two Brownian motions under $\hat{\mathbb{Q}}$ via

$$
\bar{B}_{t}^{\lambda}=\int_{0}^{t} \frac{1}{\hat{v}_{u}^{\lambda}} d \hat{B}_{A_{u}^{\lambda}}
$$

and

$$
\bar{B}_{t}^{\gamma}=\int_{0}^{t} \frac{1}{\hat{v}_{u}^{\gamma}} d \hat{B}_{A_{u}^{\gamma}} .
$$

Now note that $\mathbb{E}^{\mathbb{Q}^{\lambda}} h\left(S_{T}^{\lambda}\right)=\hat{\mathbb{E}} h\left(\hat{S}_{T}^{\lambda}\right)$ where $\hat{S}^{\lambda}$ solves

$$
\frac{d \hat{S}_{t}^{\lambda}}{\hat{S}_{t}^{\lambda}}=\hat{v}_{t}^{\lambda} d \bar{B}_{t}^{\lambda} \quad \hat{S}_{0}^{\lambda}=s_{0}
$$

and similarly for $\hat{S}^{\gamma}$, driven by $\bar{B}^{\gamma}$. For the result of the theorem to hold, we require $\hat{\mathbb{E}} h\left(\hat{S}_{T}^{\lambda}\right) \leq \hat{\mathbb{E}} h\left(\hat{S}_{T}^{\gamma}\right)$.

Using (16) and the definition of $\bar{B}^{\lambda}$ we can rewrite $\hat{S}_{T}^{\lambda}$ as

$$
\hat{S}_{T}^{\lambda}=s_{0} \exp \left(\int_{0}^{T} \hat{v}_{u}^{\lambda} d \bar{B}_{u}^{\lambda}-\frac{1}{2} \int_{0}^{T}\left(\hat{v}_{u}^{\lambda}\right)^{2} d u\right)=s_{0} \exp \left(\hat{B}_{A_{T}^{\lambda}}-\frac{1}{2} A_{T}^{\lambda}\right)
$$

and $\hat{S}_{T}^{\gamma}$ as

$$
\hat{S}_{T}^{\gamma}=s_{0} \exp \left(\hat{B}_{A_{T}^{\gamma}}-\frac{1}{2} A_{T}^{\gamma}\right) .
$$

By construction, both are functions of the same $\hat{\mathbb{Q}}$-Brownian motion $\hat{B}$. Note that given $A_{T}^{\lambda} \leq A_{T}^{\gamma}, \hat{\mathbb{E}}\left(\hat{S}_{T}^{\gamma} \mid A_{T}^{\lambda}\right)=\hat{S}_{T}^{\lambda}$. Then for convex $h$, Jensen's inequality gives

$$
\hat{\mathbb{E}}\left[h\left(\hat{S}_{T}^{\gamma}\right) \mid A_{T}^{\lambda}\right] \geq h\left(\hat{\mathbb{E}}\left(\hat{S}_{T}^{\gamma} \mid A_{T}^{\lambda}\right)\right)=h\left(\hat{S}_{T}^{\lambda}\right)
$$

giving

$$
\hat{\mathbb{E}} h\left(\hat{S}_{T}^{\gamma}\right)=\hat{\mathbb{E}}\left[\hat{\mathbb{E}}\left[h\left(\hat{S}_{T}^{\gamma}\right) \mid A_{T}^{\lambda}\right]\right] \geq \hat{\mathbb{E}} h\left(\hat{S}_{T}^{\lambda}\right)
$$

as required. 


\section{Comparisons between the $q$-optimal, minimal en- tropy and minimal martingale measures}

In order to apply our main result Theorem 3 we need to establish relationships between the market price of volatility risk process for each of the measures, given in Section 3. This section will describe such orderings, which depend crucially on the mean variance tradeoff process. We first prove a lemma which we use in our results.

Lemma 6 Let $X$ satisfy

$$
d X=\alpha\left(t, X_{t}\right) d t+\beta\left(t, X_{t}\right) d Z^{\dagger}
$$

where $Z^{\dagger}$ is a $\mathbb{P}^{\dagger}$-Brownian motion. Suppose (17) has a weak solution which is unique in law and has the Strong Markov Property. Take two realizations starting at $X_{0}=x^{(1)}, X_{0}=x^{(2)}$, with $x^{(2)}>x^{(1)}$. For all functions $m(f)$, $m: C[0, T] \rightarrow \mathbb{R}$, if

(i) $m(f)$ non-increasing in $f, \mathbb{E}^{\ddagger} m\left(X_{t}^{(2)}\right) \leq \mathbb{E}^{\ddagger} m\left(X_{t}^{(1)}\right)$,

(ii) $m(f)$ non-decreasing in $f, \mathbb{E}^{\ddagger} m\left(X_{t}^{(2)}\right) \geq \mathbb{E}^{\ddagger} m\left(X_{t}^{(1)}\right)$, where the expectation is taken with respect to measure $\mathbb{P}^{\ddagger}$, possibly different from $\mathbb{P}^{\dagger}$.

Proof: Let $x^{(2)}>x^{(1)}$ and take two independent realizations of $X$ starting at $x^{(1)}, x^{(2)}$ and denoted $X^{(1)}, X^{(2)}$. Define $\tau=\inf _{u \geq t}\left\{u: X_{u}^{(2)}=X_{u}^{(1)}\right\}$ and define

$$
\tilde{X}_{t}=\left\{\begin{array}{cc}
X_{t}^{(1)} & t \leq \tau \\
X_{t}^{(2)} & t>\tau
\end{array}\right.
$$

So $X_{t}^{(2)} \geq \tilde{X}_{t}$ by construction and $\tilde{X}_{t} \stackrel{\text { law }}{=} X_{t}^{(1)}$ since the processes are strong Markov. Then for all functions $m(f): C[0, T] \rightarrow \mathbb{R}, m(f)$ non-increasing in $f, m\left(X_{t}^{(2)}\right) \leq m\left(\tilde{X}_{t}\right)$ and $\mathbb{E}^{\ddagger} m\left(X_{t}^{(2)}\right) \leq \mathbb{E}^{\ddagger} m\left(\tilde{X}_{t}\right)=\mathbb{E}^{\ddagger} m\left(X_{t}^{(1)}\right)$ where $\mathbb{P}^{\ddagger}$ can be different to $\mathbb{P}^{\dagger}$. A similar argument is followed if $m$ is non-decreasing in $f$.

We can now prove a new observation concerning the dependence of the $q$ optimal measure on the parameter $q$. Taken together, these results enable us to compare market prices of risks for various $q$ and minimal entropy. Recall that we are assuming $g$ in (8) has sufficient continuity properties.

Theorem 7 Under stochastic volatility dynamics in $(1), \lambda^{(q)}(t, v)$ is (i) non-decreasing in $q$ if $\mu(t, v)$ is non-decreasing in $v$,

(ii) non-increasing in $q$ if $\mu(t, v)$ is non-increasing in $v$

Proof: Since $\lambda^{(q)}(t, v)=b(t, v) g^{\prime}(t, v)$, we only need consider the $q$ dependence of $g^{\prime}(t, v)=\frac{\partial g}{\partial v}(t, v)$. Consider

$$
2 \frac{\partial g}{\partial q}\left(t, v_{t}\right)=\frac{\mathbb{E}\left[\int_{t}^{T} \mu\left(u, v_{u}\right)^{2} d u e^{-\frac{q}{2} \int_{t}^{T} \mu\left(u, v_{u}\right)^{2} d u}\right]}{\mathbb{E}\left[\exp \left(-\frac{q}{2} \int_{t}^{T} \mu\left(u, v_{u}\right)^{2} d u\right)\right]}
$$


It is equivalent to examine the dependence of $\frac{\partial g}{\partial q}$ on $v$. To simplify notation, fix $t=0$ for the remainder of the proof.

The above expression may be rewritten using the mean variance tradeoff process $K_{T}=\int_{0}^{T} \mu\left(u, v_{u}\right)^{2} d u$ as

$$
2 \frac{\partial g}{\partial q}\left(0, v_{0}\right)=\frac{\mathbb{E} K_{T} e^{-\frac{q}{2} K_{T}}}{\mathbb{E} e^{-\frac{q}{2} K_{T}}} .
$$

We now try to recharacterize $K_{T}$ using ideas from Hobson [14], see his equation (17). Suppose we can find a process $\xi$, and finite constant $c$ such that

$$
\frac{q}{2} K_{T}=\int_{0}^{T} \xi\left(u, v_{u}\right) d W_{u}+\frac{1}{2} \int_{0}^{T} \xi^{2}\left(u, v_{u}\right) d u+c .
$$

Now consider $G(t, v)$ such that $G(T, v)=0$. From Itô's formula,

$$
\begin{aligned}
-G\left(0, v_{0}\right) & =\int_{0}^{T} d G\left(t, v_{t}\right) \\
& =\int_{0}^{T} G^{\prime} b\left(t, v_{t}\right) d W+\int_{0}^{T} G^{\prime} a\left(t, v_{t}\right) d t+\frac{1}{2} \int_{0}^{T} G^{\prime \prime} b\left(t, v_{t}\right)^{2} d t+\int_{0}^{T} \dot{G} d t
\end{aligned}
$$

and rearranging gives

$$
\begin{aligned}
& -\int_{0}^{T} G^{\prime} b\left(t, v_{t}\right) d W-c \\
= & -c+\left(G\left(0, v_{0}\right)+\int_{0}^{T} G^{\prime} a\left(t, v_{t}\right) d t+\frac{1}{2} \int_{0}^{T} G^{\prime \prime} b\left(t, v_{t}\right)^{2} d t+\int_{0}^{T} \dot{G} d t\right) .
\end{aligned}
$$

If we take $\xi\left(u, v_{u}\right)=G^{\prime} b\left(u, v_{u}\right)$ then we have an equation of the form (19) provided

$$
G\left(0, v_{0}\right)-c=\int_{0}^{T}\left[-\frac{q}{2} \mu\left(t, v_{t}\right)^{2}+\frac{1}{2}\left(G^{\prime}\right)^{2} b\left(t, v_{t}\right)^{2}-G^{\prime} a\left(t, v_{t}\right)-\frac{1}{2} G^{\prime \prime} b\left(t, v_{t}\right)^{2}-\dot{G}\right] d t .
$$

Thus there is a solution to (19) if we can solve

$$
\frac{q}{2} \mu\left(t, v_{t}\right)^{2}-\frac{1}{2}\left(G^{\prime}\right)^{2} b\left(t, v_{t}\right)^{2}+G^{\prime} a\left(t, v_{t}\right)+\frac{1}{2} G^{\prime \prime} b\left(t, v_{t}\right)^{2}+\dot{G}=0
$$

and $c=G\left(0, v_{0}\right)$. Using the transformation $F(t, v)=e^{-G(t, v)}$, we obtain

$$
\frac{q}{2} \mu\left(t, v_{t}\right)^{2} F=a\left(t, v_{t}\right) F^{\prime}+\frac{1}{2} F^{\prime \prime} b\left(t, v_{t}\right)^{2}+\dot{F}
$$

which is equivalent to (20). By Feynman-Kac, this has solution

$$
F(t, v)=\mathbb{E}\left[\exp \left(-\frac{q}{2} \int_{t}^{T} \mu\left(u, v_{u}\right)^{2} d u\right) \mid v_{t}=v\right]
$$

so $G(t, v)=g(t, v)$ where $g$ is given in (8). 
Now returning to the analysis of (18). Using (19) we can write

$$
e^{-\frac{q}{2} K_{T}}=e^{-\int_{0}^{T} \xi\left(u, v_{u}\right) d W-\frac{1}{2} \int_{0}^{T} \xi\left(u, v_{u}\right)^{2} d u} e^{-c}
$$

which can be written as

$$
e^{-\frac{q}{2} K_{T}}=\frac{d \tilde{\mathbb{P}}}{d \mathbb{P}} e^{-c}
$$

for a new measure $\tilde{\mathbb{P}}$ defined by

$$
\left.\frac{d \tilde{\mathbb{P}}}{d \mathbb{P}}\right|_{\mathcal{F}_{t}}=e^{-\int_{0}^{t} \xi\left(u, v_{u}\right) d W-\frac{1}{2} \int_{0}^{t} \xi\left(u, v_{u}\right)^{2} d u} .
$$

Note $\frac{d \tilde{\mathbb{P}}}{d \mathbb{P}}<e^{c}$ and thus the right hand side of $(22)$ is a true $\mathbb{P}$-martingale and $\tilde{\mathbb{P}}$ is a probability measure.

By Girsanov's theorem, under $\tilde{\mathbb{P}}, \tilde{W}_{t}=W_{t}+\int_{0}^{t} \xi\left(u, v_{u}\right) d u$ is a Brownian motion and $v$ follows the SDE

$$
d v_{t}=\left[a\left(t, v_{t}\right)-b\left(t, v_{t}\right) \xi\left(t, v_{t}\right)\right] d t+b\left(t, v_{t}\right) d \tilde{W}_{t} .
$$

From (21), the density can also be expressed in terms of $K_{T}$ and $c$ as

$$
\frac{d \tilde{\mathbb{P}}}{d \mathbb{P}}=\frac{e^{-\frac{q}{2} K_{T}}}{e^{-c}}
$$

giving $e^{-c}=\mathbb{E} e^{-\frac{q}{2} K_{T}}$. This can also be obtained directly from (19).

Returning now to the expression we are interested in (18), we can use the above expression for the new density to write

$$
2 \frac{\partial g}{\partial q}(0, v)=\mathbb{E} K_{T} \frac{d \tilde{\mathbb{P}}}{d \mathbb{P}}=\tilde{\mathbb{E}} K_{T}=\tilde{\mathbb{E}} \int_{0}^{T} \mu(u, v)^{2} d u
$$

At this point, we invoke Lemma 6 with $m(f)=\int_{0}^{T} \mu(u, f)^{2} d u$.

Corollary 8 If $\mu(t, v)$ does not depend on $v$, (so is both non-decreasing and non-increasing) then $\lambda^{(q)}$ is independent of $q$.

The following result allows us to compare minimal entropy and q-optimal market prices of risk to that of the minimal martingale measure.

Theorem 9 For a model of form (1)

(i) if $\mu(t, v)$ is non-decreasing in $v$, then $g^{\prime} \geq 0, f^{\prime} \geq 0$ and $\lambda^{(q)}=b(t, v) g^{\prime} \geq 0$, $\lambda^{(E)}=b(t, v) f^{\prime} \geq 0$;

(ii) if $\mu(t, v)$ is non-increasing in $v$, then $g^{\prime} \leq 0, f^{\prime} \leq 0$ and $\lambda^{(q)}=b(t, v) g^{\prime} \leq 0$, $\lambda^{(E)}=b(t, v) f^{\prime} \leq 0$.

Proof: (i) Apply Lemma 6 with $m(f)=\exp \left(-\frac{q}{2} \int_{t}^{T} \mu(s, f)^{2} d s\right)$ to show with two starting points $v^{(2)}>v^{(1)}$ for two processes $v_{t}^{(1)}, v_{t}^{(2)}$,

$$
g\left(t, v^{(2)}\right)=-\ln \mathbb{E} m\left(v_{t}^{(2)}\right) \geq-\ln \mathbb{E} m\left(v_{t}^{(1)}\right)=g\left(t, v^{(1)}\right)
$$

so $g^{\prime} \geq 0$ if $\mu(t, x)$ is non-decreasing in $x$. Since entropy is a limiting case of the $q$-optimal, it follows that $f^{\prime} \geq 0$ also. (ii) is similar. 
Corollary 10 If $\mu(t, v)$ does not depend on $v$, then $g^{\prime}=0, f^{\prime}=0$ and $\lambda^{(q)}=0$, $\lambda^{(E)}=0$.

These results classify the market price of volatility risks depending on the mean variance tradeoff process. The specific form for the process $v$ follows has not entered the results. Also note that for the these orderings on the market prices of volatility risks, we do not need to assume the volatility process is non-negative, this is only required for the conclusions on the option prices.

\section{Option Price Comparison under minimal martin- gale, q-optimal and minimal entropy measures}

We can now combine the results of the previous section with Theorem 3 to give some comparison results between option prices under the three methods for choosing a measure.

Theorem 11 For convex payoffs and models of the form (1) with non-negative volatility process $v$ :

(i) if $\mu(t, v)$ is non-decreasing in $v$, the minimal martingale measure option price is at least as large as q-optimal option prices (for $q \geq 1$ ), and q-optimal option prices are decreasing in $q$. In particular, option prices under the minimal martingale measure are at least as large as option prices under minimal entropy, which in turn are at least as large as option prices under the variance optimal measure.

(ii) if $\mu(t, v)$ is non-increasing in $v$, all inequalities in (i) are reversed.

Proof: Direct application of Theorems 7 and 9 along with the main Theorem 3.

Corollary 12 If $\mu(t, v)$ does not depend on $v$, option prices under the minimal martingale measure, minimal entropy measure, variance optimal measure and $q$-optimal measure (for $q>1$ ) are all equal.

This special case is a known result for the variance optimal and entropy measures, see Remark 2. However, our result also extends this to prices under the $q$-optimal measure.

\section{Comparisons under specific models of Hull-White, Heston and Stein and Stein}

Our comparison results can be used to compare option prices under different stochastic volatility models in the class (1). Popular models that we concentrate on are the Hull and White model [15], Heston model [13] and the Stein and Stein model [15].

Consider the Hull White and Heston models first. These have non-negative volatility processes, and thus fit nicely into our setup. The Hull White model 
is the simplest of the three models as it has constant coefficients. The Heston model is more complex, but despite this, we are able to do some explicit calculations with this model, illustrating some of the theoretical results.

The Hull and White model [15] specifies the square of volatility follows exponential Brownian motion. For constant $\bar{b}, \delta$, the stock $S$ and volatility $\sqrt{Y}$ follow

$$
\begin{aligned}
\frac{d S}{S} & =\mu(t, \sqrt{Y}) \sqrt{Y} d t+\sqrt{Y} d B \\
\frac{d Y}{Y} & =\bar{b} d t+\delta d W
\end{aligned}
$$

where $B$ and $W$ are independent Brownian motions. This is in the form (1) if we write $v=\sqrt{Y}$ so

$$
\frac{d S_{t}}{S_{t}}=v_{t}\left(\mu\left(t, v_{t}\right) d t+d B_{t}\right) \quad d v_{t}=\left(\frac{1}{2} \bar{b}-\frac{1}{8} \delta^{2}\right) v_{t} d t+\frac{1}{2} \delta v_{t} d W_{t} .
$$

This model is equivalent to the choice $a\left(t, v_{t}\right)=\left(\frac{1}{2} \bar{b}-\frac{1}{8} \delta^{2}\right) v_{t}$ and $b\left(t, v_{t}\right)=\frac{1}{2} \delta v_{t}$ in (1). Now volatility $v$ follows an exponential Brownian motion and hence $v_{t} \geq 0 \forall t$. The original Hull White model [15] takes $\mu\left(t, v_{t}\right)=\mu / v_{t}$, for constant $\mu$. If $\mu\left(t, v_{t}\right)=\mu$, then Remark 2 applies and option prices under the considered measures are identical. Another possible choice would be $\mu\left(t, v_{t}\right)=\mu v_{t}$.

In the Heston model [13] the squared volatility $Z$ is given by a squared Bessel process

$$
\begin{aligned}
\frac{d S}{S} & =\mu(t, \sqrt{Z}) \sqrt{Z} d t+\sqrt{Z} d B \\
d Z & =2 \kappa(m-Z) d t+2 \beta \sqrt{Z} d W
\end{aligned}
$$

with $B$ and $W$ independent Brownian motions. Of course, the original paper of Heston [13] allows for non-zero correlation, but this is beyond the scope of our current results. Writing $Z=v^{2}$ and $\bar{m}=m-\beta^{2} / 2 \kappa$ we get

$$
\frac{d S_{t}}{S_{t}}=v_{t}\left(\mu\left(t, v_{t}\right) d t+d B_{t}\right) \quad d v_{t}=\kappa\left(\bar{m} / v_{t}-v_{t}\right) d t+\beta d W_{t} .
$$

This model is equivalent to taking $a\left(t, v_{t}\right)=\kappa\left(\bar{m} / v_{t}-v_{t}\right), b\left(t, v_{t}\right)=\beta$ in (1). We assume $m>\beta^{2} / \kappa$ to guarantee non-negative $v$. If we take $\mu\left(t, v_{t}\right)=\mu v_{t}$, this is equivalent to model (H2) in Heath et al [10]. The original paper of Heston [13] takes constant drift on $S, \mu\left(t, v_{t}\right)=\mu / v_{t}$ and if a linear drift were used $\mu\left(t, v_{t}\right)=\mu$, Remark 2 would apply and option prices under the relevant measures would be equal. Each of these drift specifications will imply different relationships between option prices under various measures.

We now consider a model that has received attention in the literature, taking the Heston model with specification $\mu\left(t, v_{t}\right)=\mu v_{t}$. We are first interested in the $q$-optimal measure. It is straightforward to solve (7) in this example, the solution is of the form $g\left(t, v_{t}\right)=v_{t}^{2} h^{(q)}(t) / 2$ with $h^{(q)}(T)=0$. This gives

$$
\lambda^{(q)}(t, v)=\beta v_{t} h^{(q)}(t)
$$


with

$$
h^{(q)}(t)=\frac{\Gamma^{(q)}}{\beta^{2}} \tanh \left(\Gamma^{(q)}(T-t)+\tanh ^{-1}\left(\frac{\kappa}{\Gamma^{(q)}}\right)\right)-\frac{\kappa}{\beta^{2}}
$$

and $\Gamma^{(q)}=\sqrt{\kappa^{2}+\mu^{2} \beta^{2} q}$. This is also given in Heath et al [10] for the special case of $q=2$.

The minimal entropy market price of risk can be found similarly, and is given by

$$
\lambda^{(E)}(t, v)=\beta v_{t} h^{(1)}(t)
$$

with

$$
h^{(1)}(t)=\frac{\Gamma^{(1)}}{\beta^{2}} \tanh \left(\Gamma^{(1)}(T-t)+\tanh ^{-1}\left(\frac{\kappa}{\Gamma^{(1)}}\right)\right)-\frac{\kappa}{\beta^{2}}
$$

and $\Gamma^{(1)}=\sqrt{\kappa^{2}+\mu^{2} \beta^{2}}$. We can differentiate $\lambda^{(q)}(t, v)$ with respect to $q$, and we find it is positive. In particular, as promised by Theorem $7, \lambda^{(q)}$ is nondecreasing in $q$. A calculation also shows $h^{(q)} \geq 0$, confirming the result of Theorem 9 .

In general however, these calculations are difficult and must be repeated for each specification of drift $\mu(t, v)$. For instance, they are not straightforward in the Hull and White model. In contrast, Theorem 11 gives us general ordering results.

Curiously, the ordering implied in this example (and by the general theorem) seems at odds with the numerical result exhibited in the paper of Heath et al [10]. They compare numerically put option prices under the minimal martingale and variance optimal measures for the Heston model with the specification $\mu\left(t, v_{t}\right)=\mu v_{t}$. They find the variance optimal price to be the higher one. They also claim this relationship is reversed for call options. Both of these results are at odds with Theorem 11 and the computations in the Heston model above. Our result says the option price under the minimal martingale measure is greater than that under the variance optimal measure in this model and the relationship depends only on the convexity of the payoff function, giving the same ordering for puts and calls.

We now consider our third example, the model of Stein and Stein [26]. The volatility follows an Ornstein Uhlenbeck process with

$$
\begin{aligned}
\frac{d S}{S} & =\mu(t, \sigma) \sigma d t+\sigma d B \\
d \sigma & =-\kappa \sigma d t+\beta d W
\end{aligned}
$$

where $\kappa, \beta$ are non-negative constants and $B, W$ are independent Brownian motions. This is a special case of the more general model which has a nonzero mean reversion level. Note the volatility process $\sigma$ can be negative. This is an example of a model which does not satisfy the conditions we require for our ordering results, however, we can rewrite this model so that we may apply them, subject to some small changes to the proof of Theorem 3. Putting $v=|\sigma|$ gives the equivalent model

$$
\frac{d S_{t}}{S_{t}}=v_{t}\left(\mu\left(t, v_{t}\right) d t+d B_{t}\right) \quad d v_{t}=-\kappa v_{t} d t+\beta d W_{t}+d L
$$


where $L$ is the local time of $v$ at level zero. If $\mu(t, \sigma)=\mu \sigma$, this is a special case of model (S2) in Heath et al [10]. The original model of Stein and Stein took a constant drift $\mu(t, \sigma)=\mu / \sigma$. We cannot deal with the remaining case of a linear drift, as the transformation to a non-negative process does not allow this. This is also the reason that we have restricted to a zero mean reversion level.

Under any $\mathbb{Q} \in \mathcal{Q}$, these dynamics become

$$
\begin{aligned}
\frac{d S_{t}}{S_{t}} & =v_{t} d B_{t}^{\mathbb{Q}} \\
d v_{t} & =\left[-\kappa v_{t}-\lambda\left(t, v_{t}\right) \beta\right] d t+\beta d W_{t}^{\mathbb{Q}}+d L .
\end{aligned}
$$

Although these dynamics no longer correspond to the volatility dynamics necessary for Theorem 3 (as there is an additional local time term) to obtain the result, either a more general stochastic comparison theorem could be used, or the square of the volatility processes could be compared to order the time changes. We take the second approach and show the main result on option price ordering still applies to the restricted Stein and Stein model in (28).

Corollary 13 Under the Stein and Stein model in (28) the result of Theorem 3 holds with $v$ taking the role of the non-negative volatility process. Hence if $\lambda(t, v) \geq \gamma(t, v)$ for all $t, v$, then

$$
\mathbb{E}^{\mathbb{Q}^{\lambda}} h\left(S_{T}^{\lambda}\right) \leq \mathbb{E}^{\mathbb{Q}^{\gamma}} h\left(S_{T}^{\gamma}\right)
$$

for convex $h$.

Proof: Under two market price of risk processes $\lambda(t, v)$ and $\gamma(t, v)$ corresponding to two measures $\mathbb{Q}^{\lambda}$ and $\mathbb{Q}^{\gamma}$, the volatility processes are

$$
d v_{t}^{\lambda}=\left[-\kappa v_{t}^{\lambda}-\lambda\left(t, v_{t}^{\lambda}\right) \beta\right] d t+\beta d W_{t}^{\mathbb{Q}^{\lambda}}+d L^{v_{t}^{\lambda}}
$$

and

$$
d v_{t}^{\gamma}=\left[-\kappa v_{t}^{\gamma}-\gamma\left(t, v_{t}^{\gamma}\right) \beta\right] d t+\beta d W_{t}^{\mathbb{Q}^{\gamma}}+d L^{v_{t}^{\gamma}} .
$$

Define $\hat{\mathbb{Q}}$ as in Theorem 3, and we have

$$
d \hat{v}_{t}^{\lambda}=\left(-\kappa \hat{v}_{t}^{\lambda}-\beta \lambda\left(t, \hat{v}_{t}^{\lambda}\right)\right) d t+\beta d \hat{W}_{t}+d L^{\hat{v}_{t}^{\lambda}}
$$

and the corresponding process $\hat{v}^{\gamma}$. Introduce time changes $A_{t}^{\lambda}=\int_{0}^{t}\left(\hat{v}_{u}^{\lambda}\right)^{2} d u$ and $A_{t}^{\gamma}=\int_{0}^{t}\left(\hat{v}_{u}^{\gamma}\right)^{2} d u$ as before. As in Theorem 3, we show if $\lambda(t, v) \geq \gamma(t, v) \forall t, v \in$ $\mathbb{R}_{+}$, then $A_{T}^{\lambda} \leq A_{T}^{\gamma}$. The time change ordering is then sufficient for the option price ordering result, following the remainder of the proof of Theorem 3 . This time we show $\left(\hat{v}^{\lambda}\right)^{2} \leq\left(\hat{v}^{\gamma}\right)^{2}$ which is sufficient for the time change ordering.

Now

$$
d\left(\hat{v}_{t}^{\lambda}\right)^{2}=\left[\beta^{2}-2 \kappa\left(\hat{v}_{t}^{\lambda}\right)^{2}-2 \beta \lambda\left(t, \hat{v}_{t}^{\lambda}\right) \hat{v}_{t}^{\lambda}\right] d t+2 \beta \hat{v}_{t}^{\lambda} d \hat{W}+2 \hat{v}_{t}^{\lambda} d L^{\hat{v}_{t}^{\lambda}}
$$

where the final term is zero as local time grows only when $\hat{v}^{\lambda}=0$. Again, there is a corresponding process for $\left(\hat{v}^{\lambda}\right)^{2}$. 
We can apply a standard comparison theorem as before to give the condition $\lambda(t, v) \geq \gamma(t, v)$ for all $t$ and $v \in \mathbb{R}_{+}$.

The option price ordering result of Theorem 11 now holds for the Stein and Stein class of models provided the dynamics of $v$ in (28) are Markov, necessary for the coupling in Lemma 6 . This is the case.

Consider the restricted Stein and Stein model (28) with $\mu\left(t, v_{t}\right)=\mu v_{t}$. We can solve (7) to give

$$
\lambda^{(q)}(t, v)=\beta v_{t} h^{(q)}(t)
$$

with $h^{(q)}(t)$ defined in (25). Again, we can verify $\lambda^{(q)}$ is non-decreasing in $q$ and we know $h^{(q)} \geq 0$, confirming Theorems 7 and 9 . Taking $q=1$ gives the minimal entropy market price of risk.

The original papers of Hull and White [15] and Stein and Stein [26] take constant drifts on the stock price, $\mu(t, v)=\mu / v$. Their analysis essentially uses the minimal martingale measure by assigning market price of volatility risk zero and option prices are calculated under these assumptions. Under such a specification, the results of Theorem 11 imply the minimal martingale measure option price is the lowest of the option prices under different measures. Thus we can obtain insights into the original models and the implications of the choice of measure on option prices.

\section{Conclusion}

This paper has provided a comparison of option prices in a stochastic volatility model under the minimal martingale measure, $q$-optimal measure and minimal entropy. The results depend on a general theorem which proved option prices are decreasing in the market price of volatility risk. In the case of 'almost complete' models, we recover the well known result that option prices under each of these measures are identical. In general, the ordering of option prices under these martingale measures depends on the mean variance tradeoff, rather than the specific dynamics of the volatility process.

The stochastic volatility models in this paper have been restricted to have correlation of zero between the driving Brownian motions of the price and volatility processes. This simplification has enabled us to obtain a general ordering result on option prices and base conclusions solely on the mean variance tradeoff process. It is unlikely such a general result could be true once correlation between the stock and volatility is incorporated. However, recently some difficulties in identifying the market price of risk for these measures with non-zero correlation were overcome, so there is some hope for some progress, possibly on a case by case basis. A numerical comparison would also be possible in the general case.

Of course this analysis has concentrated on the option prices, without discussion of the hedging strategies under each approach. This is left for future research. 


\section{References}

[1] Belledin M. and C. Schlag (1999): "An Empirical Comparison of Alternative Stochastic Volatility models". Preprint, Johann Wolfgang GoetheUniversität.

[2] Biagini F, P. Guasoni and M. Prattelli (2000): "Mean Variance Hedging for Stochastic Volatility models". Mathematical Finance, 10(2), 109-123.

[3] Delbaen F. and W. Schachermayer (1996): "The Variance-Optimal martingale measure for continuous processes". Bernoulli, 2, 81-105, amendments and corrections Bernoulli, 2, 379-380.

[4] El Karoui N., M. Jeanblanc and S.E. Shreve (1998): "Robustness of the Black Scholes Formula". Mathematical Finance, 8(2), 93-126.

[5] Follmer H. and M. Schweizer (1991): "Hedging of Contingent claims under incomplete information". In Applied Stochastic Analysis, Stochastic Monographs, Vol 5, eds M.H.A Davis and R.J.Elliot, New York, Gordon and Breach, 389-414.

[6] Frey R. (1997): "Derivative Asset Analysis in models with leveldependent and stochastic volatility". CWI Quarterly, 10(1), 1-34.

[7] Frittelli M. (2000): "The minimal entropy martingale measure and the valuation problem in incomplete markets". Mathematical Finance, 10, 3952 .

[8] Gourieroux C., J.P. Laurent and H. Pham (1998): "Mean-Variance Hedging and Numeraire". Mathematical Finance, 8, 179-200.

[9] Grandits P. and T. Rheinländer (2002): "On the minimal entropy martingale measure". Annals of Probability, July.

[10] Heath D., E. Platen and M. Schweizer (2001): "A Comparison of two Quadratic Approaches to Hedging in Incomplete Markets". Mathematical Finance, 11(4), 385-413.

[11] Henderson V. (2000): "Price Comparison Results and super-replication: an application to passport options". Applied Stochastic Models in Business and Industry, 16(4), 297-310.

[12] Henderson V. and D. Hobson (2002): "Coupling and Option price comparisons in a Jump diffusion model". Oxford Finanical Research Centre Preprint 2002-MF-01.

[13] Heston S.L. (1993): "A Closed form solution for options with stochastic volatility with applications to bond and currency options". Review of Financial Studies, 6, 327-343.

[14] Hobson D. (2002): "Stochastic Volatility models, correlation and the $q$ optimal measure". Preprint, University of Bath. 
[15] Hull J.C and A. White (1987): "The Pricing of Options on Assets with Stochastic Volatilities". Journal of Finance, 42, 281-300.

[16] Karatzas I. and S. Shreve (1991): Brownian motion and stochastic calculus, Springer-Verlag New York, Second Edition.

[17] Laurent J.P and H. Pham (1999): "Dynamic programming and meanvariance hedging". Finance and Stochastics, 3, 83-101.

[18] Pham H., T. Rheinländer and M. Schweizer (1998): "Mean-variance hedging for continuous processes: new proofs and examples". Finance and Stochastics, 2(2), 173-198.

[19] Rheinländer T. (2002): "An entropy approach to stochastic volatility models". Preprint, ETH Zurich.

[20] Rheinländer T. and M. Schweizer (1997): "On $l^{2}$-projections on a space of stochastic integrals". Annals of Probability, 25(4), 1810-1831.

[21] Schöbel R. and J. Zhu (1999): "Stochastic Volatility with an OrnsteinUhlenbeck process: An extension". European Finance Review, 3, 23-46.

[22] Schweizer M. (1995): "On the minimal martingale measure and the Follmer-Schweizer decomposition". Stochastic Anal Appl, 13, 573-599.

[23] Schweizer M. (1999): "A minimality property of the minimal martingale measure". Statistics and Probability Letters, 42, 27-31.

[24] Scott L.O. (1997): "Pricing stock options in a jump diffusion model with stochastic volatility and interest rates: Application of fourier inversion methods." Mathematical Finance, 7, 413-426.

[25] Sin C. (1996): "Strictly local martingales and hedge ratios in stochastic volatility models". PhD thesis, Cornell University.

[26] Stein E.M. and J.C. Stein (1991): "Stock price distributions with stochastic volatility: an analytic approach". Review of Financial Studies, 4, 727752 . 